\title{
Factors Influencing Linezolid-Nonsusceptible Coagulase-Negative Staphylococci Dissemination Among Patients in the Intensive Care Unit: A Retrospective Cohort Study
}

\author{
Matthaios Papadimitriou-Olivgeris ${ }^{a, b}$ Nikolaos Giormezis ${ }^{c, d}$ Fotini Fligou ${ }^{b}$ \\ Apostolos Liakopoulos ${ }^{\mathrm{e}}$ Markos Marangos ${ }^{\mathrm{a}}$ Evangelos D. Anastassiou ${ }^{\mathrm{b}, \mathrm{c}}$ \\ Efthimia Petinaki ${ }^{\text {K }}$ Kriton S. Filos $^{b}$ Iris Spiliopoulou ${ }^{c, d}$ \\ ${ }^{\mathrm{a}}$ Division of Infectious Diseases and Departments of ${ }^{\mathrm{b}}$ Anaesthesiology and Critical Care Medicine and \\ 'Microbiology, School of Medicine, University of Patras, and d National Reference Laboratory for Staphylococci, \\ Patras, and ${ }^{\text {e}}$ Department of Microbiology, School of Medicine, University of Thessaly, Larissa, Greece
}

\section{Key Words}

Coagulase-negative staphylococci · Linezolid · Resistance ·

Clones $\cdot$ Intensive care unit $\cdot$ Risk factors

\begin{abstract}
Background: The aim of the present study was to identify risk factors for linezolid-nonsusceptible coagulase-negative staphylococci (CNS) dissemination in the intensive care unit. Methods: Among the 246 patients included, 33 revealed a linezolid-nonsusceptible CNS-positive culture specimen, 68 were positive for linezolid-susceptible CNS and 145 served as controls. Isolates were characterized by phenotypic and genotypic methods to species level, susceptibility to antistaphylococcal agents and clones. Results: Among the 33 linezolid-nonsusceptible CNS patients, 29 revealed Staphylococcus epidermidis and 4 Staphylococcus capitis. All S. epidermidis strains belonged to the ST22 clone (by multilocus sequence typing), 26 carried both C2534T and T2504A and 3 strains were $\mathrm{C} 2543 \mathrm{~T}$ mutations. S. capitis strains were stratified as a common pulsed-field gel electrophoresis type and carried the G2576T mutation. Risk factors for linezolid-nonsusceptible CNS isolation were linezolid administration and
\end{abstract}

mean number of linezolid-nonsusceptible CNS-positive patients in nearby beds per day. Conclusions: These results reinforce the aspect of rational antibiotic usage, but also highlight the need for strict infection control measures to prevent the dissemination of linezolid-nonsusceptible CNS.

(c) 2014 S. Karger AG, Basel

\section{Introduction}

Linezolid, a synthetic agent belonging to the oxazolidinone family, was introduced in the clinical practice in 2000 because of its specific activity against multiresistant Gram-positive cocci, especially glycopeptide-resistant enterococci and methicillin-resistant staphylococci [1]. It is a protein synthesis inhibitor binding to the $50 \mathrm{~S}$ ribosomal subunit, specifically to the peptidyl transferase center of bacteria (domain V), thus blocking the precise po-

Part of this work was presented as an oral presentation at the 23rd European Congress of Clinical Microbiology and Infectious Diseases, 27-30 April 2013, Berlin.

\section{KARGER}

E-Mail karger@karger.com www.karger.com/che (c) 2014 S. Karger AG, Basel

0009-3157/14/0596-0420\$39.50/0
Iris Spiliopoulou, MD, $\mathrm{PhD}$

Department of Microbiology, School of Medicine, University of Patras Rion, GR-26504 Patras (Greece)

E-Mail spiliopl@upatras.gr 
sitioning of t-RNA to the A-site [2]. The development of resistance to linezolid is associated with the presence of mutations in $23 \mathrm{~S}$ rRNA nucleotides, of which the most frequently reported are G2061, C2452, A2503, U2504, G2505, A2062, G2447, A2453, C2499, U2500 and G2576U [2]. Other mechanisms of linezolid resistance include mutations in genes encoding L3 and L4 ribosomal proteins and the presence of the transferable $c f r$ gene which encodes an rRNA methyltransferase [2,3]. Most reports underline the role of linezolid usage in hospitals for the emergence of resistance among Gram-positive bacteria [1-4].

Coagulase-negative staphylococci (CNS) are part of the normal skin flora. However, they cause severe infections in hospitalized patients (catheter-related bloodstream infections, endocarditis, prosthetic joint infections, etc.). Often, they are multidrug resistant, leaving as the only therapeutic options newer antimicrobials such as linezolid $[1,4,5]$. Reports from all over the world have identified low numbers of linezolid-resistant CNS and their presence is usually associated with hospitalized patients in intensive care units (ICU) [1,4-7]. Previous Greek studies have reported mutations conferring resistance to linezolid among Staphylococcus epidermidis from ICU wards of different hospitals. More specifically, the mutations T2504A and C2534T are closely associated with linezolid exposure and circulation of ST2 and ST22 clones (sequence types) $[4,8]$.

In the present study, the risk factors for developing infection or colonization with linezolid-nonsusceptible CNS during hospitalization in the adult medical/surgical ICU of a tertiary care university hospital were analyzed. The aim was to identify the resistance mechanisms of such strains and their genetic relationship in relation to antibiotic usage and other factors contributing to linezolid-nonsusceptible CNS spread in our setting.

\section{Methods}

\section{Patients}

This single-center retrospective cohort study was conducted in the adult ICU of the University Hospital of Patras, Greece, a 700bed teaching hospital, from March 2010 to February 2012. The ethics committee of the University Hospital of Patras approved the study and waived the need for informed consent (approval No. 571).

Patients with an ICU stay of at least 6 days were classified into three groups. Group 1 (case patients) comprised 33 patients with positive CNS clinical specimens (intravenous catheter tips and/or blood) that exhibited reduced susceptibility to linezolid (minimum inhibitory concentration, MIC $\geq 2 \mathrm{mg} / \mathrm{l}$ ), group 2 consisted of 68 ICU patients with intravenous catheter tip or blood cultures positive for linezolid-susceptible CNS (MIC $\leq 1 \mathrm{mg} / \mathrm{l}$ ) and patients with no CNS isolation served as group 3 (controls). Duplicate isolates of the same patient were excluded from further analysis. Bloodstream infection, catheter-related or not, was associated with systemic symptoms or signs (fever, tachycardia, tachypnea, hypotension, leukocytosis) with at least two positive blood cultures on separate occasions or a positive blood culture along with a positive culture by the same organism from an infected catheter [9]. Positive blood or catheter tip cultures not meeting the above criteria were considered as contaminants.

Phenotypic Identification and Antibiotic Susceptibility Testing Of the 33 case patients, 15 had a linezolid-susceptible CNS isolate prior to final linezolid-nonsusceptible CNS recovery. All isolates (48) were identified to species level by the Vitek 2 Advanced Expert System (bioMerieux SA, Marcy l'Étoile, France), while antibiotic susceptibility testing was performed by the disk diffusion method against the following antistaphylococcal agents: cefoxitin, tetracycline, rifampicin, gentamicin, kanamycin, erythromycin, clindamycin, fusidic acid, ciprofloxacin and sulfamethoxazole-trimethoprim [10]. MICs of oxacillin, vancomycin, linezolid and daptomycin were determined by Etest (bioMerieux) [10].

\section{Molecular Analysis}

All linezolid-nonsusceptible CNS were identified to species level by sequence analysis of the $t u f$ gene [11], whereas mutations in region $\mathrm{V}$ of $23 \mathrm{~S}$ rDNA were investigated by PCR and sequence analysis [8]. Sequence data were analyzed using Chromas (www. technelysium.com.au/chromas.html). The presence of the $c f r$ gene was tested by PCR [3]. The possible presence of mutations in ribosomal protein L4 was investigated by PCR followed by sequence analysis [12].

\section{Clonal Identification}

Clones were identified by means of SmaI DNA macrorestriction patterns and pulsed-field gel electrophoresis (PFGE) [13]. Linezolid-nonsusceptible and representative linezolid-susceptible $S$. epidermidis from different pulsotypes and phenotypes were further typed by multilocus sequence typing (http://www.mlst.net).

\section{Epidemiological ICU Data}

Epidemiological data were collected from the ICU computerized database (Criticus ${ }^{\mathrm{TM}}$, University of Patras, Greece) and patients' chart reviews. Parameters assessed included demographic characteristics (age, sex), severity scores of illness on admission, e.g. APACHE II (Acute Physiology and Chronic Health Evaluation II) score, SAPS II (Simplified Acute Physiology Score II) score, chronic illness prior to admission (e.g. diabetes mellitus, chronic obstructive pulmonary disease, chronic heart failure, chronic renal failure, malignancy), cortisone use, obesity, surgery, length of hospitalization, presence of invasive catheters, endotracheal intubation, tracheotomy, type of antibiotic administration and enteral or parenteral nutrition. Enteric colonization by vancomycin-resistant enterococci and nasal carriage of methicillin-resistant Staphylococcus aureus were also assessed by obtaining rectal and nasal samples from each patient upon admission and weekly afterwards, according to the surveillance program in the ICUs. The role of colonized or infected patients in nearby beds in dissemination of linezolid-nonsusceptible CNS strains was also investigated. Days 
Table 1. Phenotypic and genotypic characteristics of CNS isolated from the 33 study cases

\begin{tabular}{|c|c|c|c|c|c|c|c|}
\hline Species & Mutation & $\mathrm{MIC}, \mathrm{mg} / \mathrm{l}$ & PFGE & ST & $\begin{array}{l}\text { Resistance pattern } \\
\text { (disk diffusion method) }\end{array}$ & Clinical samples & $\begin{array}{l}\text { Colonizing/ } \\
\text { infecting, } \mathrm{n}\end{array}$ \\
\hline \multirow[t]{5}{*}{ S. epidermidis } & $\mathrm{C} 2534 \mathrm{~T}$ & $256(26)$ & A $(26)$ & 22 & FOX, GM, KAN, CC, FA, & blood (19) & $15 / 4$ \\
\hline & $\mathrm{T} 2504 \mathrm{~A}$ & & & & CIP, SXT & catheter tip (7) & $6 / 1$ \\
\hline & $\mathrm{C} 2534 \mathrm{~T}$ & $\begin{array}{l}2(1), \\
4(1)\end{array}$ & $\mathrm{C}(2)$ & 22 & \multicolumn{2}{|c|}{ FOX, GM, KAN, CC, CIP, SXTblood (2) } & $2 / 0$ \\
\hline & & & & & CIP, SXT, RA, E & catheter tip (2) & $2 / 0$ \\
\hline & - & & $\mathrm{D}(4)$ & 45 & $\begin{array}{l}\text { FOX, GM, KAN, CC, FA, } \\
\text { CIP, SXT, E }\end{array}$ & blood (4) & $4 / 0$ \\
\hline S. capitis & $\mathrm{G} 2576 \mathrm{~T}$ & $\begin{array}{r}6(2), \\
32(2)\end{array}$ & $\mathrm{k}(4)$ & n.a. & $\begin{array}{l}\text { FOX, GM, KAN, CC, FA, } \\
\text { CIP, SXT, RA }\end{array}$ & blood (4) & $4 / 0$ \\
\hline
\end{tabular}

Values in parentheses indicate number of isolates. MIC: linezolid MIC identified by Etest. ST = Sequence types, identified by multilocus sequence typing; $\mathrm{n} . \mathrm{a} .=$ not applicable; $\mathrm{FOX}=$ cefoxitin; $\mathrm{RA}=$ rifampicin; $\mathrm{GM}=$ gentamicin; $\mathrm{KAN}=$ kanamycin; $\mathrm{E}=$ erythromycin; $\mathrm{CC}$ = clindamycin; FA = fusidic acid; CIP = ciprofloxacin; SXT = sulfamethoxazole-trimethoprim.

at risk were defined as days in the ICU until linezolid-nonsusceptible CNS or linezolid-susceptible CNS isolation for the resistant and the susceptible study cases, respectively, and total ICU stay for the control group.

\section{Statistical Analysis}

SPSS version 19.0 (SPSS, Chicago, Ill., USA) software was used for data analyses. Categorical variables were analyzed by using the Fisher exact test or the $\chi^{2}$ test and continuous variables with the Mann-Whitney $U$ test, as appropriate. Three different univariate analyses were performed: the first comprised linezolid-nonsusceptible study cases versus controls, the second nonsusceptible study cases versus susceptible ones and, finally, susceptible study cases versus controls. Factors contributing to multicollinearity were excluded from the multivariate analysis. Backward stepwise multiple logistic regression analysis used all variables with a $\mathrm{p}<0.1$ from the univariate analysis. Odds ratios and $95 \%$ confidence intervals were calculated to evaluate the strength of any association. All statistical tests were 2 -tailed and $\mathrm{p}<0.05$ was considered statistically significant.

\section{Results}

\section{Susceptibility Testing and Molecular Analysis}

Among the 246 studied patients, 33 (13.4\%) exhibited at least 1 positive linezolid-nonsusceptible CNS culture (linezolid MIC $\geq 2 \mathrm{mg} / \mathrm{l}$ ). From this group, 29 S. epidermidis and $4 \mathrm{~S}$. capitis strains were isolated; 5 patients had bloodstream catheter-related infection (identified by clinical signs and positive blood and intravenous catheter tip culture with the same strain), while the remaining 28 cases were judged to be colonized (table 1) [9].
Overall, 26 S. epidermidis strains showing linezolid MIC $>256 \mathrm{mg} / \mathrm{l}$ carried both C2534T and T2504A mutations (Escherichia coli 23S rRNA gene numbering; table 1), while 3 carried only the C2534T mutation in the $23 \mathrm{~S}$ rDNA genes exhibiting linezolid MICs $2-4 \mathrm{mg} / \mathrm{l}$. Linezolid-resistant S. capitis strains (MICs: 6 and 32 $\mathrm{mg} / \mathrm{l}$ ) carried the G2576T mutation. No strain carried the $c f r$ gene or any mutation in the L4 ribosomal protein gene. All linezolid-nonsusceptible $S$. epidermidis strains belonged to the ST22 clone and to 3 PFGE types (A, C and $\mathrm{E}$ ). Antibiotic resistance patterns of isolates were related to their PFGE types (table 1). Linezolid-resistant $S$. capitis strains belonged to a common PFGE type, ' $\mathrm{k}$ '. From the group of 33 case patients, 15 had a linezolidsusceptible $S$. epidermidis that belonged to 2 (unrelated to A, C and E) PFGE types (B and D) and were classified as ST45 clone (table 1). All CNS were methicillin resistant. Mean MIC values among linezolid-nonsusceptible CNS were $1.7 \mathrm{mg} / \mathrm{l}$ for vancomycin (range $0.75-3 \mathrm{mg} / \mathrm{l}$ ) and $0.4 \mathrm{mg} / \mathrm{l}$ for daptomycin (range $0.064-1 \mathrm{mg} / \mathrm{l}$ ). The respective values for linezolid-susceptible CNS were 1.6 $\mathrm{mg} / \mathrm{l}$ (range $1.25-2 \mathrm{mg} / \mathrm{l}$ ) and $0.3 \mathrm{mg} / \mathrm{l}$ (range 0.19-1 $\mathrm{mg} / \mathrm{l})$.

\section{Risk Factors for Colonization or Infection by Linezolid-Nonsusceptible CNS}

Risk factors associated with linezolid-nonsusceptible CNS colonization or infection from the univariate analyses are shown in table 2 , whereas results from multivariate 
Table 2. Univariate analysis for risk factors for linezolid-nonsusceptible CNS isolation during ICU hospitalization

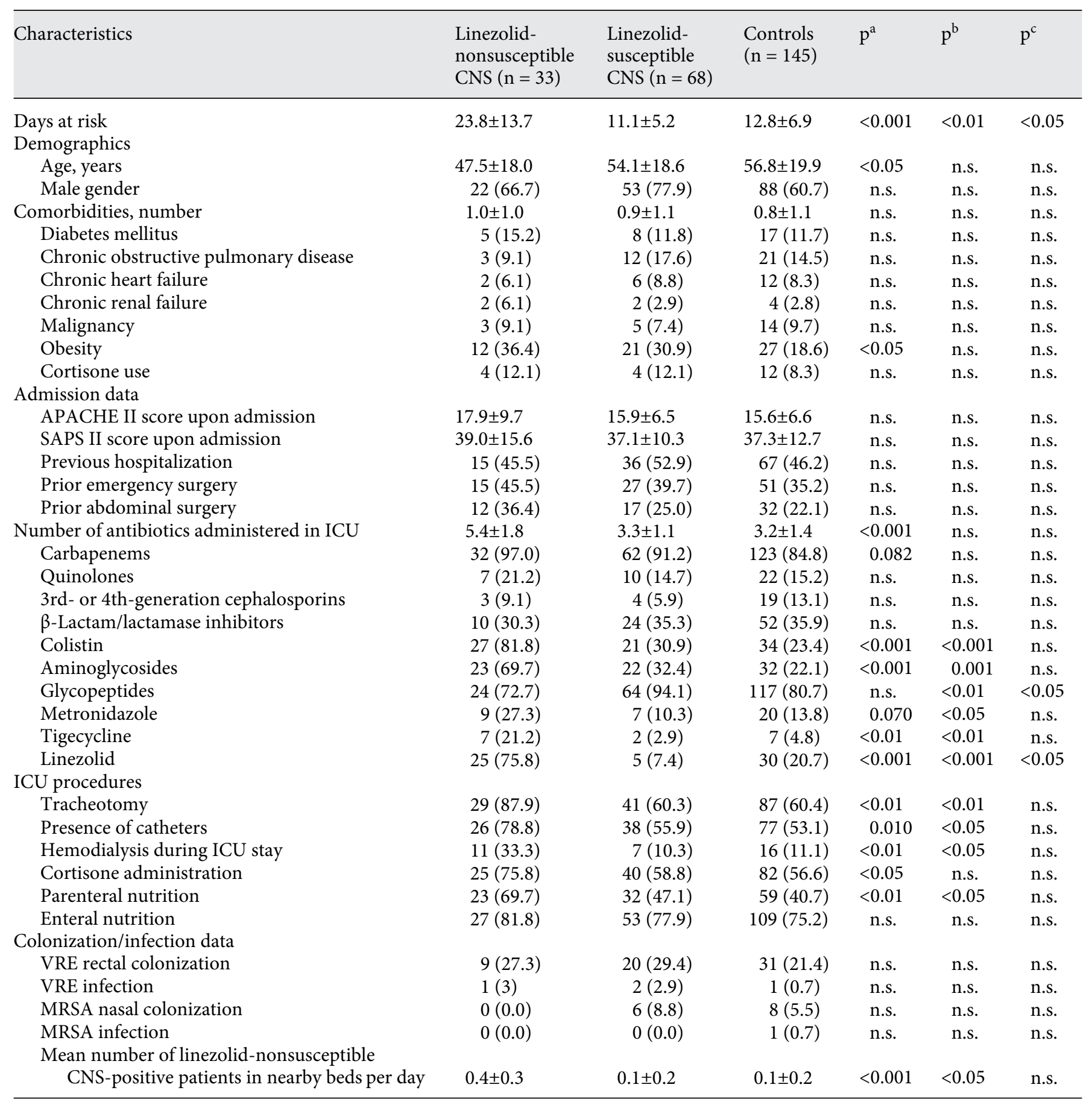

Data are presented as number of patients (with percentages in parentheses) or means \pm SD. Colistin: only IV administration of colistin is included. Presence of catheters: all patients after ICU admission were intubated, mechanically ventilated and continuously monitored with a central venous catheter, an arterial catheter and a urinary catheter. Presence of catheters does not include the aforementioned catheters. VRE = Vancomycin-resistant enterococcus; MRSA = methicillin-resistant S. aureus; n.s. = nonsignificant.

${ }^{a}$ Comparison between linezolid-nonsusceptible CNS patients vs. controls. ${ }^{b}$ Comparison between linezolid-nonsusceptible CNS patients vs. linezolid-susceptible CNS patients. ${ }^{c}$ Comparison between linezolid-susceptible CNS patients vs. controls. 
Table 3. Multivariate analysis of risk factors for linezolid-nonsusceptible CNS isolation during ICU stay

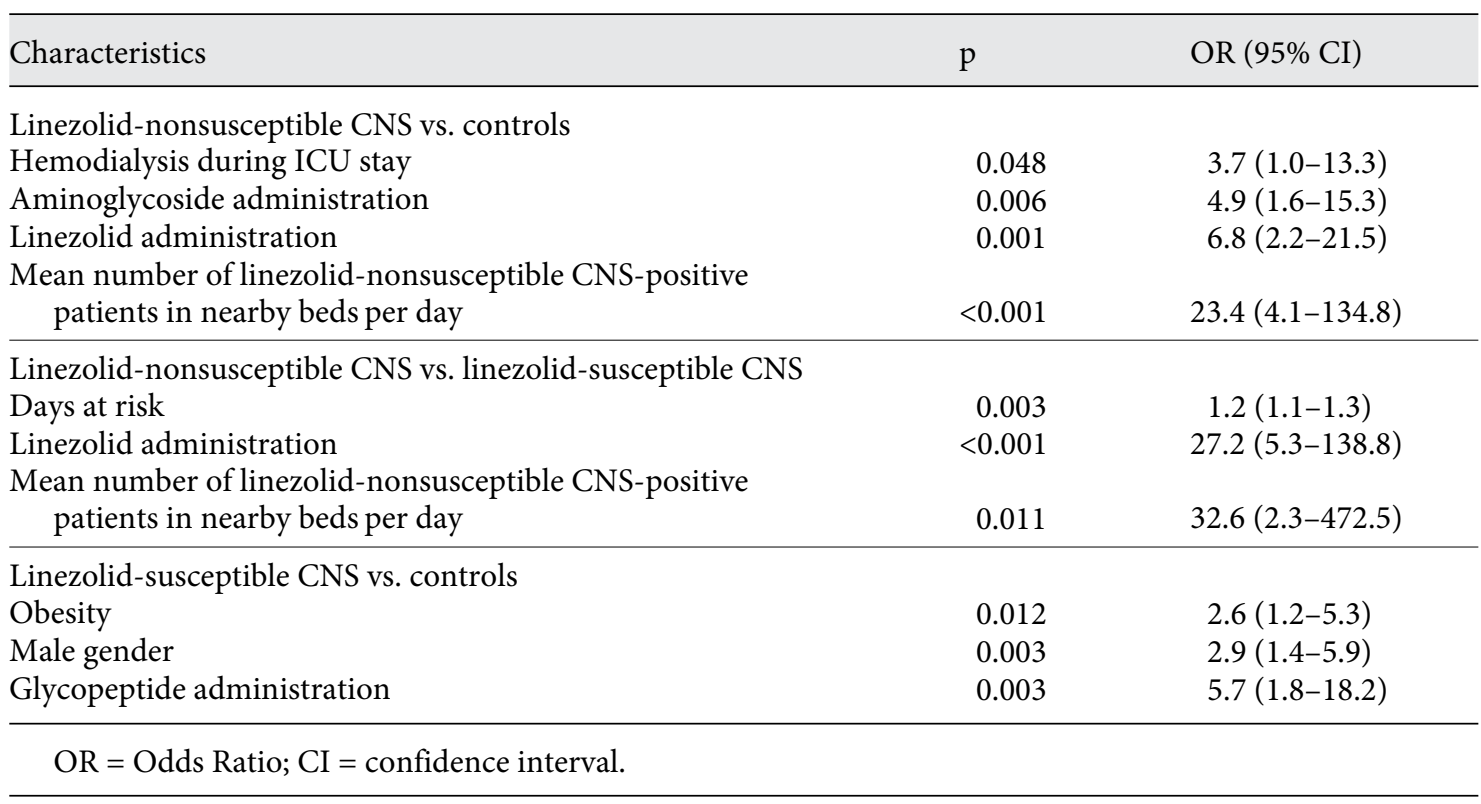

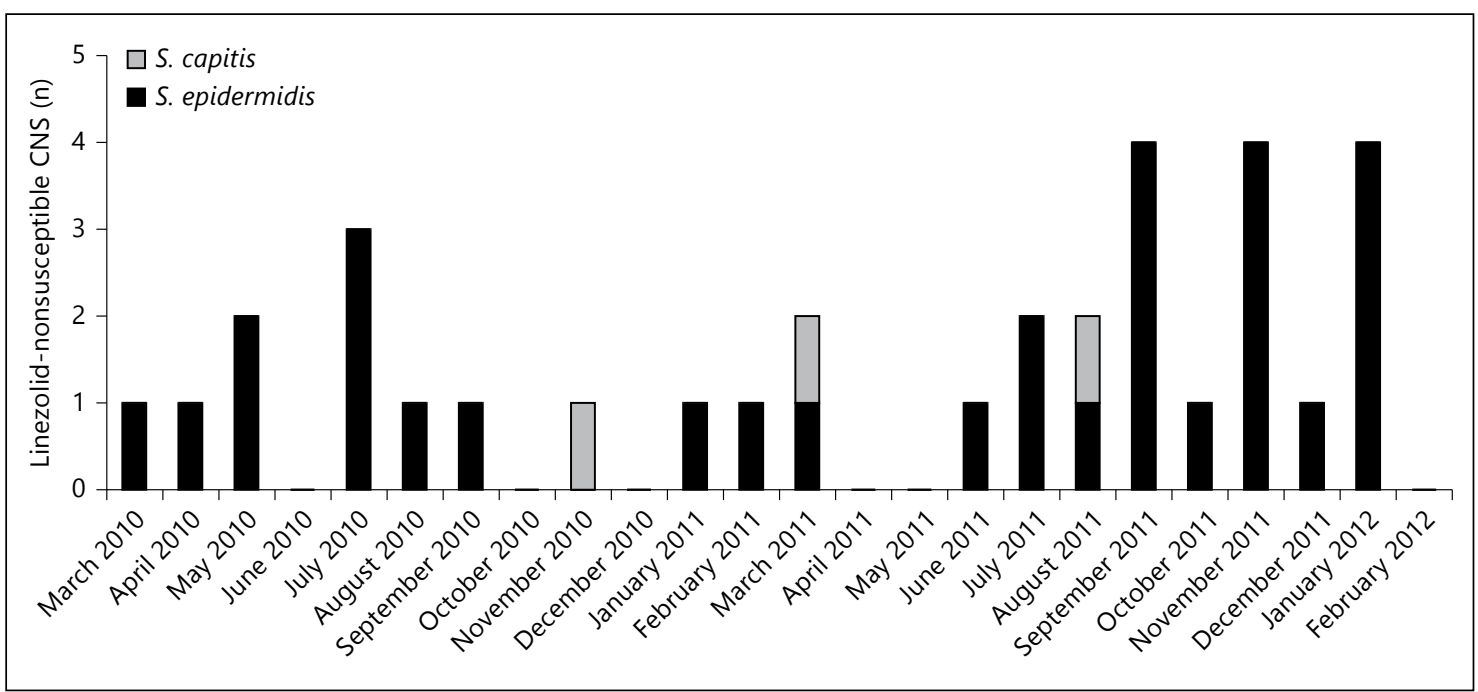

Fig. 1. Isolation of linezolid-nonsusceptible CNS per month.

analyses appear in table 3. Compared to controls, hemodialysis during ICU stay, aminoglycoside and/or linezolid administration and number of linezolid-nonsusceptible CNS-positive patients in nearby beds per day were significantly associated with linezolid-nonsusceptible CNS isolation. Multivariate analysis of linezolid-nonsusceptible CNS-positive and linezolid-susceptible CNS-positive patients revealed that days at risk, linezolid administration and number of linezolid-nonsusceptible CNS-posi- tive patients in nearby beds per day were significantly higher in the first group. Obesity, male gender and glycopeptide administration were independently associated with isolation of linezolid-susceptible CNS compared to controls. No statistically significant differences were detected in the univariate analysis between infected and colonized patients by linezolid-nonsusceptible CNS.

Figure 1 shows the number of new linezolid-nonsusceptible CNS cases per month. During the last 12 months 
of the study (March 2011 to February 2012), 21 out of 112 patients (18.8\%) were linezolid-nonsusceptible CNSpositive compared to 12 out of 124 (9.7\%) during the first 12 months $(\mathrm{p}=0.038)$.

\section{Discussion}

The widespread use of linezolid due to the reduced activity of glycopeptides against Gram-positive cocci has led to the emergence and dissemination of linezolid resistance. Recently, Gu et al. [1] reported a total of 351 linezolid-resistant CNS cases from Europe, North America, South America and Asia (53.6, 42.5, 2.8 and $1.1 \%$, respectively). All isolates were resistant to methicillin, as well as to a wide range of antistaphylococcal agents, underlying the need for accurate detection of resistance to linezolid and judicious use of the drug, since prolonged treatment induces resistance [1]. The isolates of the present study were also methicillin resistant, with antibiotic resistance patterns correlating to their PFGE types. Previous studies in Greece, including strains from our institution, identified linezolid-resistant CNS carrying C2534T, G2576T and T2504A mutations, the latter of which is associated with high levels of linezolid MIC $[4,8]$. This fact is also verified in the present study, where 26 cases of $S$. epidermidis expressing high levels of linezolid MIC carried both C2534T and T2504A mutations. Furthermore, the S. epidermidis ST22 clone seems to circulate steadily in our setting [4]. Although 3 S. epidermidis strains were linezolid susceptible (MIC: $2-4 \mathrm{mg} / \mathrm{l}$ ), they were positive for the presence of the C2534T mutation. In a study by Ferreira et al. [14] among 101 staphylococci phenotypically tested, the range of linezolid MIC was $0.5-4 \mathrm{mg} / \mathrm{l}$, but no molecular analysis was performed. In another study, linezolid nonsusceptibility among staphylococci was associated with the G2576T mutation in $23 \mathrm{~S} \mathrm{rDNA}$, whereas 5 isolates carried the mobile $c f r$ element [15]. Additionally, the presence of the G2576T mutation in 23S rDNA (mutations in L3 or L4 genes) resulted in reduced susceptibility to linezolid among enterococci $[16,17]$.

Importantly, we report for the first time that linezolidresistant S. capitis strains bear the G2576T mutation and belong to a common PFGE type. Resistance to linezolid among $S$. capitis was also identified in China due to the presence of a novel C2104T mutation [18].

Linezolid administration is reported to be one of the most important risk factors for linezolid-resistant Grampositive cocci isolation in hospital outbreaks $[4-7,19]$.

Linezolid-Resistant Staphylococci in the ICU
However, it should be noted that there are not enough data regarding the risk factors contributing to the emergence of linezolid-resistant CNS [4-7].

In our studied group, 8 out of 33 patients did not receive linezolid for at least 6 months prior to admission and during their ICU stay, suggesting that linezolid administration, although important, is not by itself the only factor contributing to the spread of linezolid-resistant pathogens. Additionally, ICUs remain the source of silent dissemination; therefore, the determination of risk factors involved in potential linezolid-resistant CNS outbreaks in such settings provides essential information for understanding the emergence and evolution of future outbreaks [4-6].

Furthermore, even though it has been previously suggested $[4,6,19]$, this is the first study proving, with multivariate analysis, that the presence of linezolidnonsusceptible CNS-positive patients in nearby beds plays an important role in the dissemination of these pathogens.

Increased length of stay was also found to be an important risk factor for the evolution and isolation of linezolid-nonsusceptible CNS. As mentioned in other studies regarding multidrug-resistant pathogens, being hospitalized near an already colonized patient usually increases the chances of acquiring the microorganism. Prolonged ICU stay predisposes to infection, especially when patients receive linezolid as an anti-Gram-positive coverage $[20,21]$.

An interesting finding was that colistin and aminoglycosides administration was identified by univariate analysis as a risk factor for the isolation of linezolid-nonsusceptible CNS compared to patients with linezolid-susceptible isolates and controls (table 2). This result can be explained by the fact that colistin and aminoglycosides are administered in combination with linezolid to patients not responding to carbapenems and glycopeptides. Therefore, no correlation exists between colistin or aminoglycoside administration and the spread of CNS resistant to linezolid.

It is noteworthy that all linezolid-nonsusceptible CNS belonged to common clones (ST22 for S. epidermidis and pulsotype $\mathrm{k}$ for $S$. capitis). Also, susceptible strains from the same patients belonged to different PFGE and sequence types (ST45), suggesting a clonal-related dissemination of linezolid-resistant strains $[4,6]$. However, since different mutations were simultaneously detected among these strains, a continuous evolution of multidrug-resistant bacteria also occurs under the pressure of antibiotic usage among critically ill ICU patients. The number of 
infected patients by linezolid-nonsusceptible CNS was low in the present study, as also reported by Potoski et al. [19].

\section{Conclusions}

Besides linezolid usage, patient-to-patient transmission of linezolid-nonsusceptible CNS occurs among ICU patients, suggesting the necessity for strict infection control measures such as adequate central venous catheter handling and hand hygiene of health care workers.

\section{Acknowledgments}

The study was carried out under the permission of the ethics committee of the University Hospital of Patras (No. 571).

This research was partially supported by funding from the National Reference Laboratory for Staphylococci, Greece, under the scientific responsibility of I.S. and E.D.A. (grant C954, KEELPNO).

\section{Disclosure Statement}

The authors have no conflicts of interest.

\section{References}

$1 \mathrm{Gu}$ B, Kelesidis T, Tsiodras S, Hindler J, Humphries RM: The emerging problem of linezolid-resistant Staphylococcus. J Antimicrob Chemother 2013;68:4-11.

$\checkmark 2$ Long KS, Vester B: Resistance to linezolid caused by modifications at its binding site on the ribosome. Antimicrob Agents Chemother 2012;56:603-612.

$\checkmark 3$ Mendes RE, Deshpande LM, Castanheira M, DiPersio J, Saubolle MA, Jones RN: First report of $c f r$-mediated resistance to linezolid in human staphylococcal clinical isolates recovered in the United States. Antimicrob Agents Chemother 2008;52:2244-2246.

4 Liakopoulos A, Spiliopoulou I, Damani A, Kanellopoulou M, Schoina S, Papafragas E, Marangos M, Fligou F, Zakynthinos E, Makris D, Protonotariou E, Tsiapara F, Filos K, Diza E, Anastassiou ED, Petinaki E: Dissemination of two international linezolid-resistant Staphylococcus epidermidis clones in Greek hospitals. J Antimicrob Chemother 2010;65:1070-1071.

5 Treviño M, Martínez-Lamas L, Romero-Jung PA, Giráldez JM, Alvarez-Escudero J, Regueiro BJ: Endemic linezolid-resistant Staphylococcus epidermidis in a critical care unit. Eur J Clin Microbiol Infect Dis 2009;28:527-533.

6 Kelly S, Collins J, Maguire M, Gowing C, Flanagan M, Donnelly M, Murphy PG: An outbreak of colonization with linezolid-resistant Staphylococcus epidermidis in an intensive therapy unit. J Antimicrob Chemother 2008; 61:901-907.

7 Seral C, Sáenz Y, Algarate S, Duran E, Luque P, Torres C, Castillo FJ: Nosocomial outbreak of methicillin- and linezolid-resistant Staphylococcus epidermidis associated with catheterrelated infections in intensive care unit patients. Int J Med Microbiol 2011;301:354-358.
Liakopoulos A, Neocleous C, Klapsa D, 15 Mendes RE, Jones RN, Deshpande LM, Ross Kanellopoulou M, Spiliopoulou I, Mathiopoulos KD, Papafrangas E, Petinaki E: A T2504A mutation in the 23S rRNA gene responsible for high-level resistance to linezolid of Staphylococcus epidermidis. J Antimicrob Chemother 2009;64:206-207.

-9 Horan TC, Andrus M, Dudeck MA: CDC/ NHSN surveillance definition of health careassociated infection and criteria for specific types of infections in the acute care setting. Am J Infect Control 2008;36:309-332.

10 CLSI: Performance Standards for Antimicro- 17 Spiliopoulou I, Damani A, Chini V, Zerva L, bial Susceptibility Testing: Twenty-First Informational Supplement, M100-S21. Wayne, Clinical and Laboratory Standards Institute, 2011.

$\checkmark 11$ Kontos F, Petinaki E, Spiliopoulou I, Maniati M, Maniatis AN: Evaluation of a novel method based on PCR restriction fragment length polymorphism analysis of the tuf gene for the identification of Staphylococcus species. J Microbiol Methods 2003;55:465-469.

12 Wong A, Reddy SP, Smyth DS, AgueroRosenfeld ME, Sakoulas G, Robinson DA: Polyphyletic emergence of linezolid-resistant staphylococci in the United States. Antimicrob Agents Chemother 2010;54:742-748.

13 Tenover FC, Arbeit RD, Goering RV, Mickelsen PA, Murray BE, Persing DH, Swaminathan B: Interpreting chromosomal DNA restriction patterns produced by pulsed-field gel electrophoresis: criteria for bacterial strain typing. J Clin Microbiol 1995;33:2233-2239.

14 Ferreira AM, Bonesso MF, Mondelli AL, Camargo $\mathrm{CH}$, Cunha MLRS: Oxacillin resistance and antimicrobial susceptibility profile of Staphylococcus saprophyticus and other staphylococci isolated from patients with urinary tract infections. Chemotherapy 2012;58: 482-491. JE, Sader HS: Daptomycin activity tested against linezolid-non susceptible Gram-positive clinical isolates. Microb Drug Resist 2009; 15:245-249.

16 Chen H, Wu W, Ni M, Liu Y, Zhang J, Xia F, He W, Wang Q, Wang Z, Cao B, Wang H: Linezolid-resistant clinical isolates of enterococci and Staphylococcus cohnii from a multicentre study in China: molecular epidemiology and resistance mechanisms. Int J Antimicrob Agents 2013;42:317-321.

Kolonitsiou F, Anastassiou ED, Petinaki E: Linezolid-resistant enterococci in Greece: epidemiological characteristics. Chemotherapy 2011;57:181-185.

18 Cai JC, Hu YY, Zhang R, Zhou HW, Chen GX: Linezolid-resistant clinical isolates of methicillin-resistant coagulase-negative staphylococci and Enterococcus faecium from China. J Med Microbiol 2012;61:1568-1573.

19 Potoski BA, Adams J, Clarke L, Shutt K, Linden PK, Baxter C, Pasculle AW, Capitano B, Peleg AY, Szabo D, Paterson DL: Epidemiological profile of linezolid-resistant coagulase-negative staphylococci. Clin Infect Dis 2006;43:165-171.

20 Safdar N, Maki DG: The commonality of risk factors for nosocomial colonization and infection with antimicrobial-resistant Staphylococcus aureus, enterococcus, Gram-negative bacilli, Clostridium difficile, and Candida. Ann Intern Med 2002;136:834-844.

21 Petinaki E, Kanellopoulou M, Damani A, Foka A, Spiliopoulou I, Skalmoutsou N, Raitsiou B, Valakis K, Papafragas E: Linezolid-resistant Staphylococcus cohnii, Greece. Emerg Infect Dis 2009;15:116-118. 